\title{
Exploring Fluctuations and Phase Equilibria in Fluid Mixtures via Monte Carlo Simulation
}

\author{
Alan R Dentont and Michael P Schmidt \\ Department of Physics, North Dakota State University, Fargo, ND 58108-6050, USA
}

\begin{abstract}
Monte Carlo simulation provides a powerful tool for understanding and exploring thermodynamic phase equilibria in many-particle interacting systems. Among the most physically intuitive simulation methods is Gibbs ensemble Monte Carlo (GEMC), which allows direct computation of phase coexistence curves of model fluids by assigning each phase to its own simulation cell. When one or both of the phases can be modeled virtually via an analytic free energy function [M. Mehta and D. A. Kofke, Mol. Phys. 79, 39 (1993)], the GEMC method takes on new pedagogical significance as an efficient means of analyzing fluctuations and illuminating the statistical foundation of phase behavior in finite systems. Here we extend this virtual GEMC method to binary fluid mixtures and demonstrate its implementation and instructional value with two applications: (1) a lattice model of simple mixtures and polymer blends and (2) a free-volume model of a complex mixture of colloids and polymers. We present algorithms for performing Monte Carlo trial moves in the virtual Gibbs ensemble, validate the method by computing fluid demixing phase diagrams, and analyze the dependence of fluctuations on system size. Our opensource simulation programs, coded in the platform-independent Java language, are suitable for use in classroom, tutorial, or computational laboratory settings.

PACS numbers: 64.60.De, 61.20.Ja, 61.20.Gy, 64.75.Xc, 01.40.-d
\end{abstract}

$\dagger$ Corresponding author. Electronic address: alan.denton@ndsu.edu 


\section{Introduction}

Phase transitions and critical phenomena are topics fundamental to most undergraduate and graduate courses in thermodynamics and statistical mechanics [1]. Aside from their intrinsic interest and practical relevance, phase transitions provide a rich conceptual context for charting the path from a microscopic Hamiltonian to macroscopic properties of materials via partition functions and free energies. Over the past sixty years, molecular simulations have provided important insights into the thermodynamic phase behavior of systems ranging from simple atomic fluids to complex macromolecular materials. Monte Carlo and molecular dynamics methods, in particular, have clarified the subtle interplay between energy and entropy in determining stability of competing phases [2, 3]. Monte Carlo simulation has been further exploited as an aid in teaching statistical mechanics [4, 5, 6].

Computer simulations of many-particle systems can readily identify mechanically stable (metastable) states, corresponding to local minima in the free energy of the system as a function of externally controlled parameters. For fluid systems, external parameters may include temperature, pressure, density, and (in the case of mixtures) concentration. More difficult is finding the global minimum in the free energy, which is required to establish thermodynamic stability. The task is especially challenging near a first-order phase transition, where two bulk phases (e.g., vapor and liquid in a one-component system, or $A$-rich and $B$-rich phases in a mixture of $A$ and $B$ species) may coexist in equilibrium.

In simulations of finite model systems, the free energy cost associated with interfaces between phases results in hysteresis and tends to inhibit the simultaneous presence of more than one phase in a single simulation cell [2]. For this reason, mapping out thermodynamic phase diagrams using computer simulation traditionally involves computing the free energy, usually via thermodynamic integration of the internal energy, and then performing a coexistence analysis via a Maxwell common-tangent construction. An alternative approach, which has been applied to both fluid and magnetic systems, is histogram reweighting [6].

A more direct and intuitive route to analyzing phase equilibria and calculating densities of coexisting phases is the Gibbs ensemble Monte Carlo (GEMC) method. Introduced by Panagiotopoulos [7, 8, 9, 10], this method models each phase in its own simulation cell. By elegantly avoiding both the complication of interfaces and the need for thermodynamic integration, the GEMC method provides a computationally efficient and conceptually transparent means of computing fluid phase diagrams. The GEMC method has been widely applied as a research tool to analyze phase behavior of simple fluids and fluid mixtures [8], as well as complex fluids, such as colloid-polymer mixtures [10, 11, 12] and charge-stabilized colloidal suspensions [13]. To date, however, the pedagogical potential of the GEMC method has been relatively less appreciated.

In this paper, we explore the GEMC method as a tool for introducing students to the statistical nature of fluctuations and phase behavior in finite systems. We are inspired by a useful variation of the method, proposed by Mehta and Kofke [14, which incorporates a 
thermodynamic model via an analytic free energy or equation of state (EOS). By modeling one of the phases explicitly and the other as virtual (via an EOS), the GEMC-EOS method gains efficiency over the original GEMC method and is reliable when the EOS of the virtual phase is accurately known.

The GEMC-EOS method was conceived and tested for one-component systems and applied to a simple fluid of particles interacting via Lennard-Jones pair potentials. A simplified, pedagogically appealing version of the GEMC-EOS method models both phases as virtual [14]. The purpose of the present work is to extend this "virtual GEMC method" to fluid mixtures and to demonstrate the value of the method in exploring and elucidating the statistical nature of demixing.

The remainder of the paper is organized as follows. In Sec. 2, we briefly review the GEMC-EOS method and then describe our extension to fluid mixtures. In Sec. 3, we demonstrate the application of the virtual GEMC method to the demixing behavior of two model systems: (1) a lattice model of mixtures, and (2) a free-volume model of colloidpolymer mixtures. For the latter, we analyze density fluctuations as functions of system size and proximity to the critical point. Finally, in Sec. 4, we conclude by emphasizing the significance of the virtual GEMC method as a classroom and computational laboratory tool.

\section{Methods}

\subsection{Gibbs Ensemble Monte Carlo Simulation}

By allowing distinct simulation boxes to exchange particles and volume, but not otherwise interact, the Gibbs ensemble Monte Carlo method can efficiently equilibrate coexisting phases (see Fig.11). Each box accommodates one of the phases at equal temperatures, pressures, and chemical potentials, with no need for interfaces. Originally introduced by Panagiotopoulos [7] to model liquid-vapor coexistence of simple one-component fluids, the GEMC method was later extended to mixtures [8]. Since its introduction, the GEMC method has been refined and used to map out phase diagrams for a wide variety of systems [9, 10].

Trial moves in Monte Carlo simulations of thermal systems are typically accepted with probabilities consistent with the condition of detailed balance [2]. According to this condition, the average rate of transition from an old $(o)$ to a new $(n)$ state equals, in equilibrium, the average reverse transition rate:

$$
P(o) \pi(o \rightarrow n)=P(n) \pi(n \rightarrow o),
$$

where $P(o)$ and $P(n)$ are probabilities of finding the system in states $o$ and $n$ and $\pi(o \rightarrow n)$ is the transition probability between $o$ and $n$. Assuming that transitions $o \rightarrow n$ and $n \rightarrow o$ are attempted at equal rates, the classic Metropolis algorithm [15] imposes Eq. (11) by accepting trial moves with probability [2, 3]

$$
\mathcal{P}(o \rightarrow n)=\min \left\{1, \frac{P(n)}{P(o)}\right\} \text {. }
$$



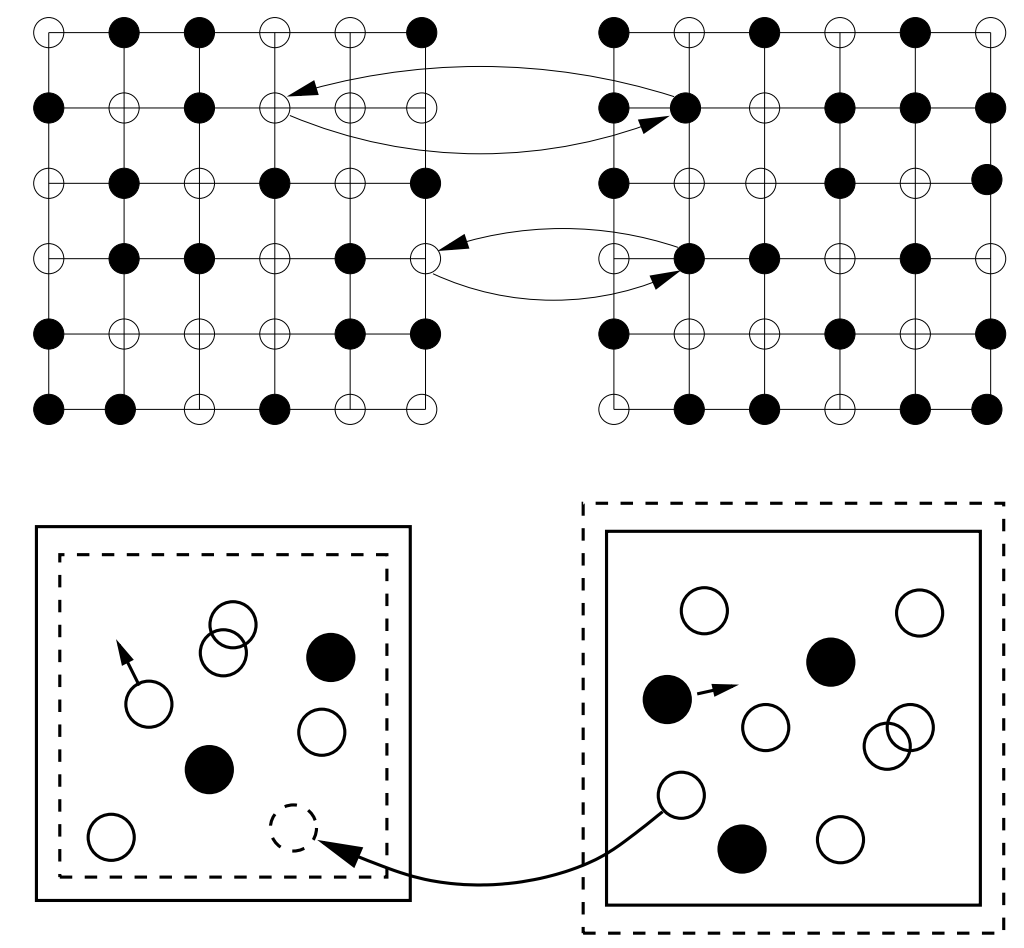

Figure 1. Schematic illustration of trial moves performed in the Gibbs ensemble Monte Carlo method. Top: particle transfers between two boxes in the lattice model of binary mixtures (Sec. 3.1). Bottom: particle displacements, volume changes, and particle transfers in a model of colloid-polymer mixtures (Sec. 3.2). The virtual GEMC method replaces both of the boxes by a virtual phase described by a free energy.

In the canonical (constant- $N V T$ ) Gibbs ensemble, a microstate of a binary mixture, at absolute temperature $T$, is specified by the positions of all particles (collectively denoted by $\{\mathbf{r}\})$, the volume $V_{i}$ of each box $(i=1,2)$, under the constraint of constant total volume $V=V_{1}+V_{2}$, and the particle numbers $N_{i j}$ of the two species $(j=A, B)$ in each box, with constant total numbers $N_{1}=N_{A 1}+N_{B 1}$ and $N_{2}=N_{A 2}+N_{B 2}$. The probability distribution for the possible microstates is given by

$$
P\left(\left\{N_{i j}\right\},\left\{V_{i}\right\}, T ;\{\mathbf{r}\}\right) \propto P_{\text {id }} e^{-\beta U(\{\mathbf{r}\})},
$$

where $\beta \equiv 1 /\left(k_{B} T\right), U=U_{1}+U_{2}$ is the internal energy of both boxes, $U_{i}$ being the internal energy of box $i$, and

$$
P_{\text {id }}\left(\left\{N_{i j}\right\},\left\{V_{i}\right\}\right) \propto \frac{V_{1}^{N_{1}} V_{2}^{N_{2}}}{N_{A 1} ! N_{B 1} ! N_{A 2} ! N_{B 2} !}
$$

is the probability distribution for an ideal mixture.

\subsection{Virtual Gibbs Ensemble Monte Carlo: Modeling Phases by a Free Energy}

The GEMC-EOS method replaces one of the simulation boxes by a virtual phase containing no explicit particles, but represented instead by a thermodynamic model in the form of a 
free energy or equation of state. In the virtual GEMC method, both phases are modeled as virtual with a prescribed excess free energy $F_{\mathrm{ex}}$, associated with interparticle interactions. The probability density for a configuration in which box $i$ has volume $V_{i}$ and contains $N_{i j}$ particles of species $j$ is then given by [cf. Eq. (3)]

$$
P \propto P_{\mathrm{id}} e^{-\beta F_{\mathrm{ex}}} .
$$

According to Eqs. (2) and (5), the acceptance probability for a trial move from an old $(o)$ to a new $(n)$ state is given by the ratio of the corresponding probability densities:

$$
\mathcal{P}(o \rightarrow n)=\min \left\{1, \frac{P_{\mathrm{id}}(n)}{P_{\mathrm{id}}(o)} e^{-\beta \Delta F_{\mathrm{ex}}}\right\},
$$

where $\Delta F_{\text {ex }}=F_{\text {ex }}(n)-F_{\text {ex }}(o)$ is the associated change in excess free energy. From Eq. (44), a trial transfer of volume $\Delta V$ from phase 1 to phase $2\left(V_{1} \rightarrow V_{1}-\Delta V, V_{2} \rightarrow V_{2}+\Delta V\right)$ is accepted with minimum probability

$$
\mathcal{P}_{\text {vol }}=\left(1-\frac{\Delta V}{V_{1}}\right)^{N_{1}}\left(1+\frac{\Delta V}{V_{2}}\right)^{N_{2}} e^{-\beta \Delta F_{\mathrm{ex}}} .
$$

In practice, trial moves in $\ln \left(V_{1} / V_{2}\right)$ improve efficiency, with acceptance probability [2, 3]

$$
\mathcal{P}_{\text {vol }}^{\prime}=\left(1+\frac{\Delta V}{V_{1}}\right)^{N_{1}+1}\left(1-\frac{\Delta V}{V_{2}}\right)^{N_{2}+1} e^{-\beta \Delta F_{\mathrm{ex}}} .
$$

Finally, a trial transfer of a particle, say of species $A$ from phase 1 to $2\left(N_{A 1} \rightarrow N_{A 1}-1\right.$, $\left.N_{A 2} \rightarrow N_{A 2}+1\right)$ is accepted with minimum probability

$$
\mathcal{P}_{\text {trans }}=\frac{V_{2}}{V_{1}} \frac{N_{A 1}}{N_{A 2}+1} e^{-\beta \Delta F_{\text {ex }}} .
$$

In the next section, to illustrate the utility of the virtual GEMC method in exploring demixing phenomena, we apply the method to two model mixtures.

\section{Applications of the Virtual GEMC Method to Demixing}

\subsection{Lattice Model of Binary Mixtures}

A simple lattice model provides an instructive introduction to phase separation. Consider a lattice of $N$ sites, each occupied by a particle of type $A$ or $B$ with volume fractions defined as $\phi_{A} \equiv N_{A} / N$ and $\phi_{B} \equiv N_{B} / N$, the total volume fraction being conserved: $\phi_{A}+\phi_{B}=1$ (see Fig. 1). The entropy of mixing is given by

$$
S_{\text {mix }}=k_{B} \ln \left(\frac{N !}{N_{A} ! N_{B} !}\right) \simeq-k_{b}\left[N_{A} \ln \phi_{A}+N_{B} \ln \phi_{B}\right],
$$

using Stirling's approximation, $\ln N ! \simeq N \ln N-N$ (valid for $N \gg 1$ ). Suppose now that only nearest-neighbor pairs interact and that correlations between particles can be neglected. 


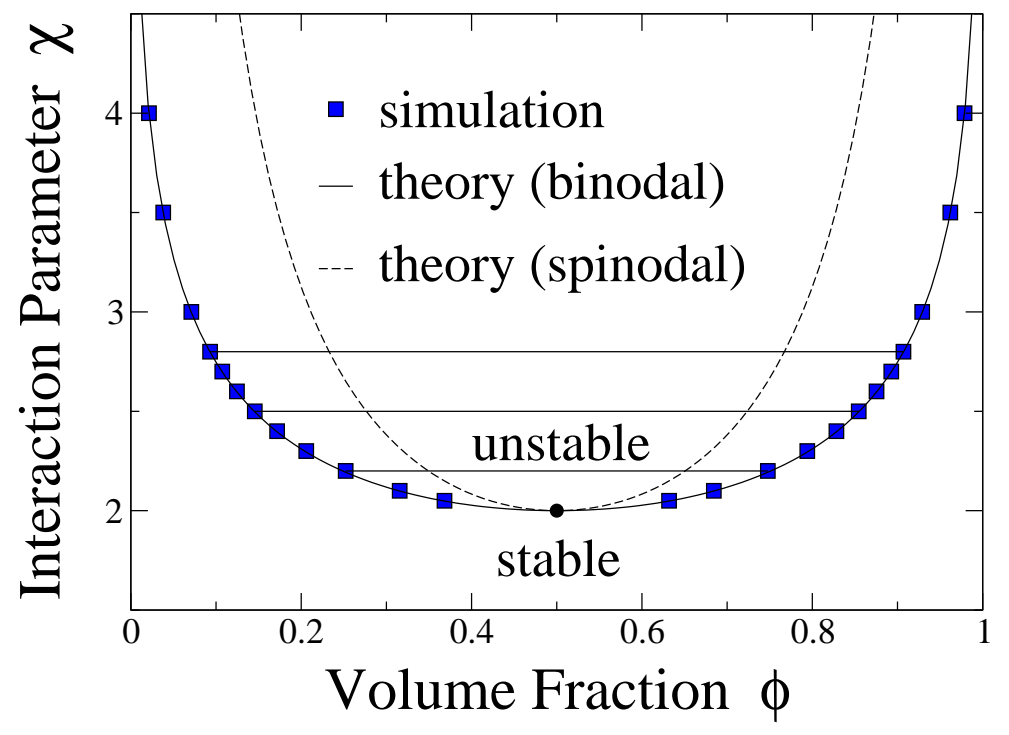

Figure 2. Phase diagram for the lattice mixture model. Squares: data from virtual GEMC simulations with both phases described by the mixing free energy of Eq. (13). Solid and dashed curves: exact binodal and spinodal curves [Eqs. (14), (15)]. Circle: theoretical critical point. Representative tie lines join coexisting phases on the binodal.

In this mean-field approximation, a particle of type $i$ occupies a given site with probability $\phi_{i}$. The internal energy, then independent of configuration, can be expressed as

$$
U=\frac{1}{2}\left(N_{A} \phi_{A} \epsilon_{A A}+N_{B} \phi_{B} \epsilon_{B B}\right)+N_{A} \phi_{B} \epsilon_{A B},
$$

where $\epsilon_{i j}$ denotes the interaction energy between a pair of particles of species $i$ and $j$. The corresponding internal energy of mixing is

$$
U_{\text {mix }}=U-\frac{1}{2}\left(N_{A} \epsilon_{A A}+N_{B} \epsilon_{B B}\right)=\chi N_{A} \phi_{B},
$$

where $\chi \equiv \epsilon_{A B}-\left(\epsilon_{A A}+\epsilon_{B B}\right) / 2$ is the Flory interaction parameter, which characterizes the incompatibility of the two species. Finally, with $\phi \equiv \phi_{A}$, the Helmholtz mixing free energy, $F_{\text {mix }} \equiv U_{\text {mix }}-T S_{\text {mix }}$, is approximated (per site) by

$$
\frac{\beta F_{\mathrm{mix}}}{N}=\phi \ln \phi+(1-\phi) \ln (1-\phi)+\chi \phi(1-\phi) \text {. }
$$

The phase behavior of the lattice model predicted by the above mean-field theory is easily deduced from the analytic expression for the free energy [Eq. (13]. In equilibrium, two phases coexist at equal temperatures, pressures, and chemical potentials, $\mu_{A}$ and $\mu_{B}$, of the two species. Incompressibility of the lattice and conservation of total volume fraction reduce the coexistence criteria to a single condition: $\mu_{A}=\partial F_{\text {mix }} / \partial \phi=0$. The coexistence curve (binodal) thus takes the analytic form

$$
\chi=\frac{1}{2 \phi-1} \ln \left(\frac{\phi}{1-\phi}\right) \text {. }
$$


The inflection points of the free energy define the spinodal curve,

$$
\frac{\partial^{2} F_{\mathrm{mix}}}{\partial \phi^{2}}=0 \Rightarrow \chi=\frac{1}{2 \phi(1-\phi)},
$$

inside of which the mixture is thermodynamically unstable and spontaneously demixes. The binodal and spinodal curves terminate at a lower critical point, $\phi_{\text {crit }}=0.5, \chi_{\text {crit }}=2$, below which the mixture is stable. Figure 2 shows the resulting phase diagram, where the interaction parameter $\chi$ plays the role of an inverse temperature.

In the virtual GEMC method, each phase is governed by the mean-field free energy of Eq. (13). With total volume fraction conserved, the only independent trial moves are exchanges of particles between the two boxes. A trial exchange of an $A$ particle in box 1 for a $B$ particle in box 2 , for example, is accepted with probability

$$
\mathcal{P}_{\text {exch }}=e^{-\beta \Delta F_{\text {mix }}}=\frac{N_{A 1} N_{B 2}}{\left(N_{A 2}+1\right)\left(N_{B 1}+1\right)} e^{-\beta \Delta U_{\text {mix }}},
$$

where $F_{\text {mix }}$ is the total mixing free energy and

$$
\Delta U_{\text {mix }}=2 \chi\left(\phi_{1}-\phi_{2}-1 / N_{1}\right)
$$

with $\phi_{i} \equiv N_{A i} / N_{i}$. For sufficiently large systems, Eq. (16) can be approximated by

$$
\mathcal{P}_{\text {exch }}=\frac{\phi_{1}\left(1-\phi_{2}\right)}{\phi_{2}\left(1-\phi_{1}\right)} e^{-\beta \Delta U_{\text {mix }}} .
$$

The Boltzmann factor in Eqs. (16) and (18) evidently favors demixing if $\chi>0$, while the entropic prefactor always favors mixing. The competition is decided by the magnitude of $\chi$.

As an illustration of the GEMC method with both phases treated virtually, we have implemented Eq. (18) for the lattice model and performed simulations to calculate several points on the coexistence curve (binodal). A trial move figuratively flips a coin (i.e., generates a random number) to decide, with equal probabilities, whether to exchange an $A$ particle for a $B$ particle, or a $B$ for an $A$, between the two boxes. Choosing $N_{1}=N_{2}=1000$, and initializing with equal numbers of $A$ and $B$ particles in each box, we equilibrated the system for $10^{4} \mathrm{MC}$ steps, a step being defined as a trial exchange of every particle. We then accumulated data over the next $10^{4}$ steps to calculate average values of $\phi_{A 1}$ and $\phi_{A 2}$ over a range of $\chi$ values. Because virtual phases have no explicit particles to displace, these simulations are extremely fast, with typical CPU times of a few minutes on a PC, scaling linearly with system size.

Our numerical results, plotted in Fig. 2, agree essentially exactly with the analytic expression for the binodal [Eq. (14)], thus validating the virtual GEMC method. On approaching the critical point, we observed growth of fluctuations and frequent switching of phases between the two boxes. Phase switching is easily suppressed, however, by increasing the number of particles. In a tutorial setting, students could, for example, probe miscibility in different regions of the phase diagram and explore the dependence of fluctuations on system size and proximity to the critical point. For this purpose, it is valuable that the binodal and spinodal curves are also known analytically. 


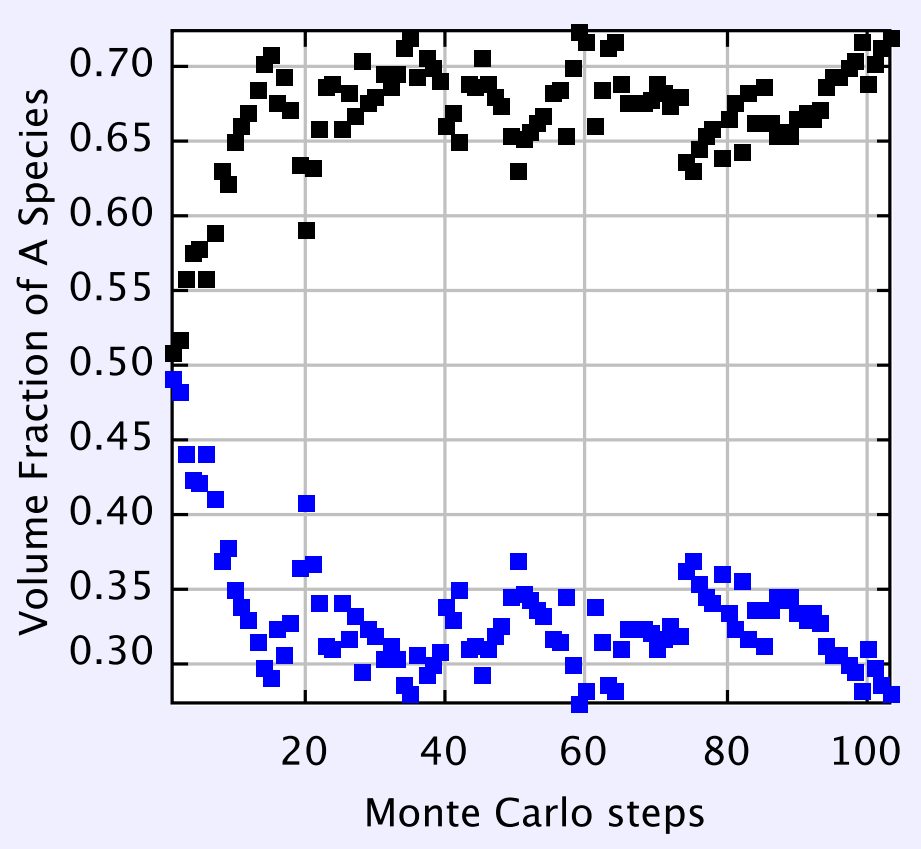

Figure 3. Typical diagnostic data from a virtual GEMC simulation of the lattice mixture model [Eq. (13)] : cumulative average volume fraction of $A$ species in each box vs. number of Monte Carlo steps during equilibration for interaction parameter $\chi=2.1$.

To facilitate pedagogical applications on a variety of platforms, we have coded our simulations in the Java programming language using the Open Source Physics (OSP) library [18, 19]. The graphical user interface provided by the AbstractSimulation class in the controls package of the OSP library allows the user to easily input parameters and start, stop, and step through a simulation. Figure 3 shows typical diagnostic data, collected during the equilibration stage, displayed with the OSP frames package. Our code can be easily made compatible, however, with any graphics library.

A closely related model depicts a polymer blend as a mixture of chains of lengths (degrees of polymerization) $M_{A}$ and $M_{B}$, whose segments (monomers) fully occupy the sites of a lattice. Connecting monomers to form chains reduces their entropic free energy by a factor of inverse chain length. Making an analogous mean-field approximation, the FloryHuggins theory for this model yields [17]

$$
\frac{\beta F_{\text {mix }}}{N}=\frac{\phi}{M_{A}} \ln \phi+\frac{1-\phi}{M_{B}} \ln (1-\phi)+\chi \phi(1-\phi),
$$

where $N_{A}$ now represents the number of $A$ chains (rather than $A$ segments) and $\phi=$ $N_{A} M_{A} / N$. In the symmetric case $\left(M_{A}=M_{B}=M\right)$, the phase diagram is identical to Fig. 2, but with $\chi$ replaced by $M \chi$ on the vertical axis. Asymmetric blends $\left(M_{A} \neq M_{B}\right)$ display much richer phase behavior. The Flory-Huggins mixing free energy [Eq. (19)] could be used to explore, via the virtual GEMC method, demixing of polymer blends. 


\subsection{Colloid-Polymer Mixtures}

As a second application of the virtual GEMC method, we consider a widely studied system from soft matter physics - a suspension of colloidal particles mixed with free (nonadsorbing) polymers [20, 22]. The classic Asakura-Oosawa-Vrij (AOV) model [23, 24] idealizes the colloidal particles as hard spheres, monodisperse in radius $R_{c}$, and the polymer coils as effective spheres with a radius $R_{p}$ equal to the average radius of gyration. The polymers are modeled as mutually ideal (noninteracting), but impenetrable to the colloids, with which they have hard-sphere interactions. Although real polymer coils fluctuate in size [25], the AOV model portrays the effective polymer spheres as monodisperse in size (see Fig. 1). The size ratio $q=R_{p} / R_{c}$ is then the one model parameter that distinguishes different mixtures.

The thermodynamic state of the system is specified by the total volume $V$ and numbers of colloids and polymers, $N_{c}$ and $N_{p}$, with respective number densities $\rho_{c}=N_{c} / V$ and $\rho_{p}=N_{p} / V$ and volume fractions $\phi_{c}=(4 \pi / 3) \rho_{c} R_{c}^{3}$ and $\phi_{p}=(4 \pi / 3) \rho_{p} R_{p}^{3}$. In the Gibbs ensemble, the particle numbers in the two boxes are denoted $N_{c 1}, N_{c 2}, N_{p 1}, N_{p 2}$, and constrained by $N_{c 1}+N_{c 2}=N_{c}$ and $N_{p 1}+N_{p 2}=N_{p}$. With only hard interparticle interactions, the thermodynamic state is independent of temperature, there being no energy scale. For contact with experiments, it is helpful to imagine the system in osmotic equilibrium with a reservoir of pure polymer, which exchanges polymers with the system to maintain fixed polymer chemical potential. The reservoir density $\rho_{p}^{r}$ plays the role of an inverse temperature.

To describe the phase behavior of the AOV model of colloid-polymer mixtures, Lekkerkerker et al [26] have developed a free-volume theory by expressing the Helmholtz free energy density (to within a constant) as

$$
f\left(\phi_{c}, \phi_{p}\right)=k_{B} T \rho_{c}\left(\ln \phi_{c}-1\right)+f_{h s}\left(\phi_{c}\right)+f_{p}\left(\phi_{c}, \phi_{p}\right) .
$$

The first two terms on the right side are the colloid ideal-gas and hard-sphere excess free energy densities, the latter being accurately approximated by the Carnahan-Starling relation [28]:

$$
\beta f_{h s}\left(\phi_{c}\right)=\rho_{c} \frac{\phi_{c}\left(4-3 \phi_{c}\right)}{\left(1-\phi_{c}\right)^{2}} .
$$

If colloid-polymer correlations are neglected (mean-field approximation), the polymer free energy density can be approximated by that of an ideal gas of polymers confined to the free volume, i.e., the volume not excluded by the hard-sphere colloids:

$$
f_{p}\left(\phi_{c}, \phi_{p}\right)=k_{B} T \rho_{p}\left[\ln \left(\frac{\phi_{p}}{\alpha\left(\phi_{c}\right)}\right)-1\right] \text {. }
$$

Here $\alpha\left(\phi_{c}\right)$ is the free-volume fraction of polymers amidst colloids, which is reasonably approximated by scaled-particle theory:

$$
\alpha\left(\phi_{c}\right)=\frac{1}{1-\phi_{c}} \exp \left(-\sum_{m=1}^{3} C_{m} \gamma^{m}\right),
$$




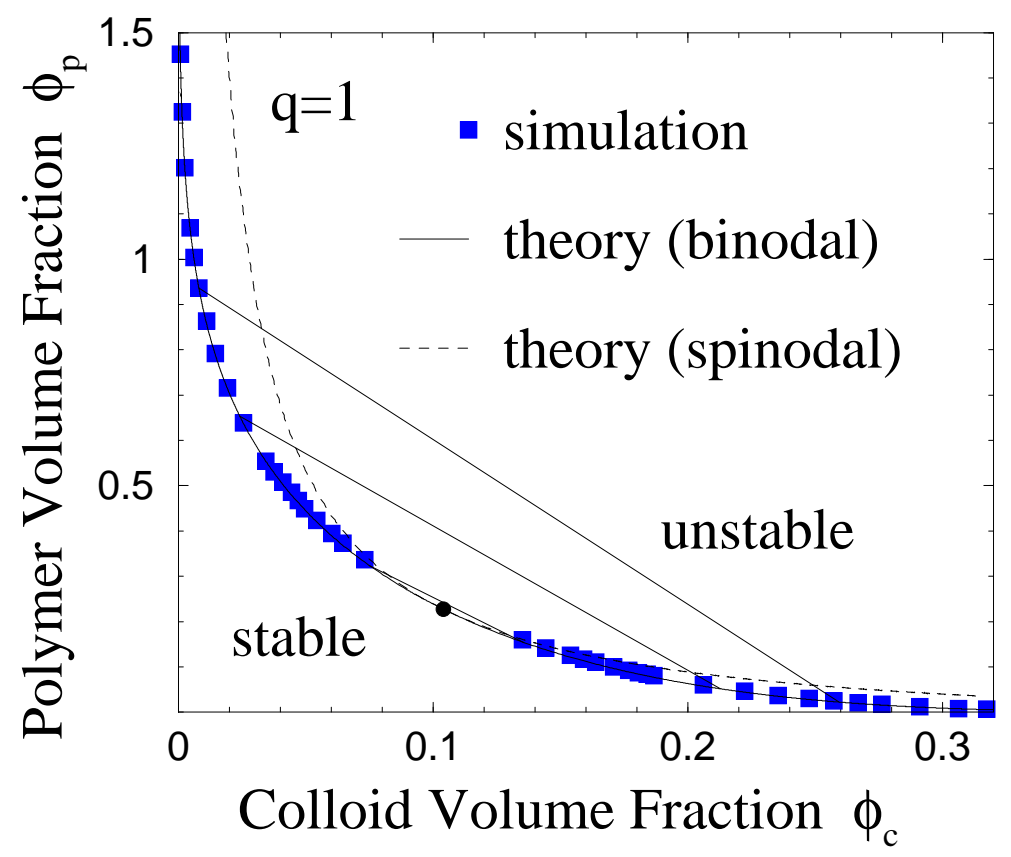

Figure 4. Phase diagram for the AOV model of colloid-polymer mixtures with size ratio (polymer radius of gyration over colloid radius) $q=1$. Squares: data from virtual GEMC simulations with both phases described by free energy from free-volume theory. Solid and dashed curves: binodal and spinodal curves from Maxwell common-tangent construction. Circle: theoretical critical point. Tie lines join coexisting phases on the binodal.

where $\gamma=\phi_{c} /\left(1-\phi_{c}\right), C_{1}=3 q+3 q^{2}+q^{3}, C_{2}=\left(9 q^{2} / 2\right)+3 q^{3}$, and $C_{3}=3 q^{3}$. For ideal polymers, equality of polymer chemical potentials in the system and reservoir implies $\phi_{p}=\phi_{p}^{r} \alpha\left(\phi_{c}\right)$, where $\phi_{p}^{r}=(4 \pi / 3) \rho_{p}^{r} R_{p}^{3}$ is the volume fraction of the polymer reservoir.

Given the analytic expression for the free energy [Eqs. (20)-(23)], it is straightforward to explore phase stability of the AOV model predicted by the mean-field free-volume theory. The demixing binodal can be calculated from a Maxwell construction that equates pressures and chemical potentials of coexisting phases, while the spinodal is defined by the inflection points of the free energy. Figure 4 shows the resulting fluid demixing phase diagram for mixtures with a size ratio $q=1$, for which the polymer coils are impenetrable to the colloids. The colloid-rich and colloid-poor branches of the binodal correspond, respectively, to colloidal "liquid" and "vapor" phases.

For simulations, the free energy expressed by Eqs. (20)-(23) can be recast in the form

$$
f\left(\phi_{c}, \phi_{p}\right)=f_{\text {id }}\left(\phi_{c}, \phi_{p}\right)+f_{\text {ex }}\left(\phi_{c}, \phi_{p}\right)
$$

comprising an ideal-gas free energy density,

$$
\beta f_{\text {id }}\left(\phi_{c}, \phi_{p}\right)=\rho_{c}\left(\ln \phi_{c}-1\right)+\rho_{p}\left(\ln \phi_{p}-1\right),
$$


and an excess free energy density

$$
\beta f_{\mathrm{ex}}\left(\phi_{c}, \phi_{p}\right)=\beta f_{h s}\left(\phi_{c}\right)-\rho_{p} \ln \alpha\left(\phi_{c}\right) .
$$

The acceptance probabilities for the trial moves in the virtual Gibbs ensemble are given by Eqs. (8) and (9) combined with Eq. (26). A transfer of volume $\Delta V$ from phase 1 to 2 is accepted with probability

$$
\mathcal{P}_{\text {vol }}^{\prime}=\left(1-\frac{\Delta V}{V_{1}}\right)^{N_{1}+1}\left(1+\frac{\Delta V}{V_{2}}\right)^{N_{2}+1} e^{-\beta \Delta F_{\mathrm{ex}}},
$$

while transferring a colloid from phase 1 to 2 is accepted with probability,

$$
\mathcal{P}_{\text {trans }}=\frac{V_{2}}{V_{1}} \frac{N_{c 1}}{N_{c 2}+1} e^{-\beta \Delta F_{\text {ex }}} .
$$

The last factor on the right side of Eqs. (27) and (28) may be expressed as

$$
e^{-\beta \Delta F_{\mathrm{ex}}}=\left(\frac{\alpha_{1}(n)}{\alpha_{1}(o)}\right)^{N_{p 1}}\left(\frac{\alpha_{2}(n)}{\alpha_{2}(o)}\right)^{N_{p 2}} e^{-\beta \Delta F_{\mathrm{hs}}} .
$$

To illustrate our implementation of the virtual GEMC method for the AOV model, we have performed simulations of mixtures with a size ratio of $q=1$. For efficiency, we pre-computed $f_{h s}$ and $\alpha$ vs. $\phi_{c}$ and stored these data in a lookup table. Initializing the system with equal numbers of particles and equal volumes in the two boxes, we fixed the total number of colloids at $N_{c}=2000$, chose the volume to give an average colloid volume fraction of $\phi_{c}=0.1$, and adjusted the polymer volume fraction by varying the total number of polymers over a range $2500<N_{p}<10^{4}$. After equilibrating for $10^{4}$ steps, we accumulated statistics over the next $10^{4}$ steps to calculate average volume fractions of each species in each box. A step is here defined as one trial volume exchange and a trial transfer of every particle. In attempting a particle transfer, we first randomly chose a box (1 or 2), then randomly chose a species of particle to be transferred to the other box.

The results of our simulations are plotted on the demixing phase diagram in Fig. 4. Each run generated a pair of points on the binodal - one on the liquid branch and one on the vapor branch. Theory and simulation again agree very closely, further validating the method. As with the lattice model, students could, in a computational laboratory setting, explore phase stability in different parts of the phase diagram and explore the variation of fluctuations with system size and proximity to the critical point. Although the free-volume theory of the AOV model does not yield the binodal and spinodal curves in analytic form, the calculation of these curves by a Maxwell construction on the free energy is straightforward.

Figure 5 shows a typical diagnostic trace near the beginning of a run in the unstable region, revealing significant fluctuations in the densities of the coexisting phases. To analyze these variations, it is helpful to define the root-mean-square (rms) fluctuations

$$
\sigma_{i} \equiv \sqrt{\frac{1}{N_{M C}} \sum_{j=1}^{N_{M C}}\left(\phi_{i}^{(j)}-\phi_{i}\right)^{2}}
$$




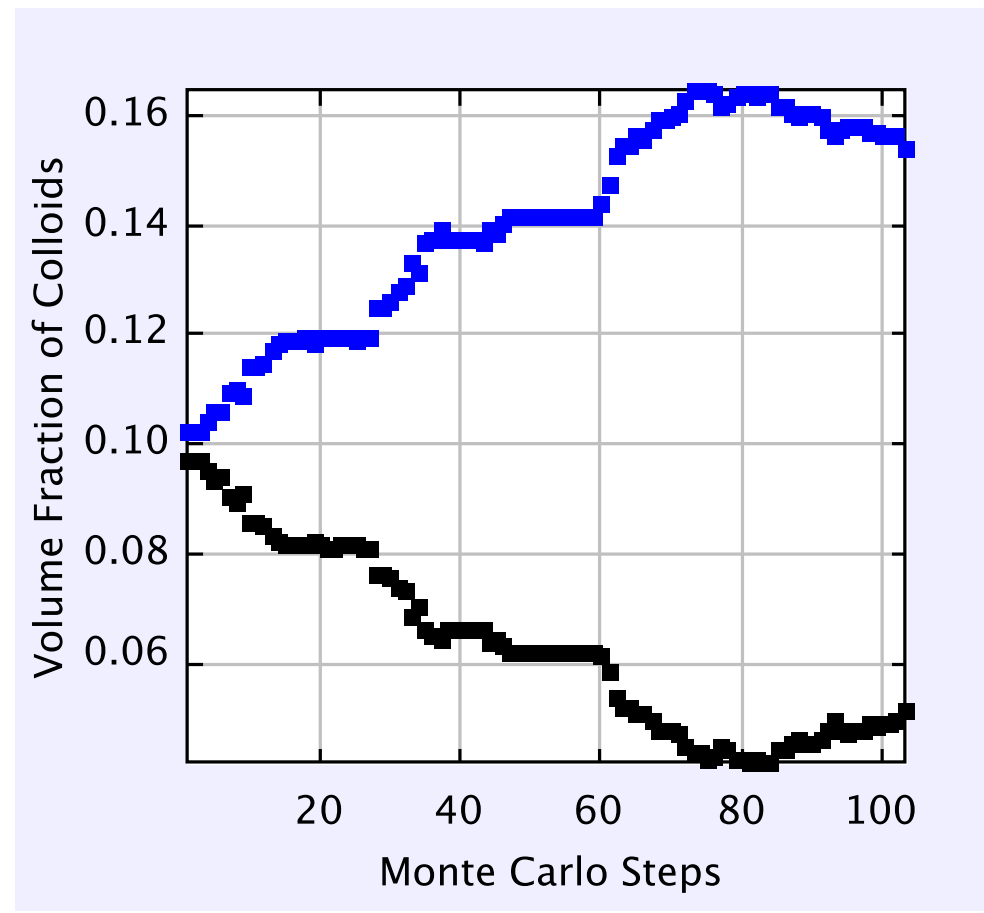

Figure 5. Typical diagnostic data from virtual GEMC simulation of AOV model of colloidpolymer mixtures: cumulative average volume fractions of colloids in each box vs. Monte Carlo steps during equilibration for size ratio $q=1$ and total volume fractions $\phi_{c}=0.1$, and $\phi_{p}=0.3$.

where $\phi_{i}$ is the mean volume fraction of species $i(i=c, p)$ and $\phi_{i}^{(j)}$ is the volume fraction of species $i$ sampled at Monte Carlo step $j$. Figure 6 shows the relative rms fluctuations, $\sigma_{c} / \phi_{c}$ and $\sigma_{p} / \phi_{p}$, for two sets of systems - one relatively small, with colloid number $N_{c}=100$ and total polymer numbers in the range $N_{p}=6 \times 10^{2}-10^{3}$, and the other 10 times larger, with $N_{c}=1000$ and $N_{p}=6 \times 10^{3}-10^{4}$ - computed from runs of $2 \times 10^{5} \mathrm{MC}$ steps. To minimize correlations between successive samples of the volume fraction, we spaced the samples by intervals of 1000 steps.

As seen in panel (a) of Fig. 6, fluctuations grow upon approaching the critical point and as the system size is decreased. Panel (b) replots the data from panel (a), but with fluctuations in the larger systems now scaled by a factor of $\sqrt{10}$ (square-root of system size ratio). The collapse of the two data sets upon scaling demonstrates the well-known result that relative fluctuations scale with the inverse-square-root of the particle number: $\sigma_{i} / \phi_{i} \sim 1 / \sqrt{N}$. As an exercise, students could test this scaling property by performing simulations over a range of system size. 

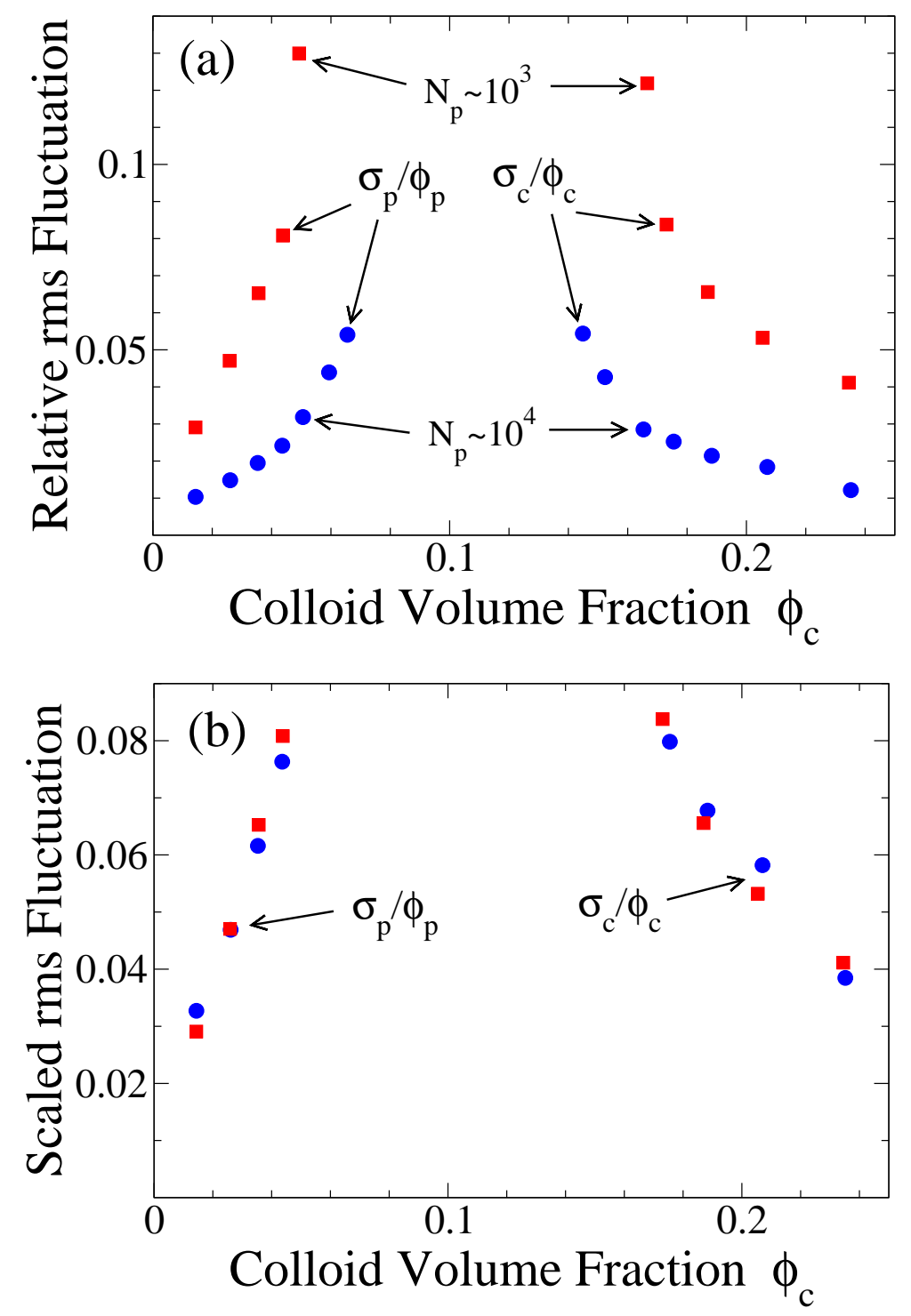

Figure 6. Root-mean-square (rms) fluctuations in densities of coexisting phases, corresponding to the phase diagram in Fig. 4. (a) Relative fluctuations $\sigma_{c} / \phi_{c}$ and $\sigma_{p} / \phi_{p}$ for systems with polymer numbers in the range $N_{p}=6 \times 10^{2}-10^{3}$ (squares) and for systems with 10 times as many particles, $N_{p}=6 \times 10^{3}-10^{4}$ (circles). (b) Same data, but with larger-system fluctuations now scaled by a factor of $\sqrt{10}$. 


\section{Concluding Remarks}

In summary, we have extended to fluid mixtures a version of the Gibbs ensemble Monte Carlo simulation method that models both phases virtually via a thermodynamic equation of state or free energy. As illustrations, we have applied the method to demixing in two models for which, in a mean-field approximation, analytic free energy expressions are known: a lattice model of simple mixtures and a free-volume model of colloid-polymer mixtures. For both models, we have validated the method by computing fluid demixing phase diagrams that closely agree with those calculated from Maxwell common-tangent constructions.

As a computational method, virtual GEMC has two main virtues. First, for finite systems whose equation of state may be known approximately from experiment, but whose interparticle interactions remain unknown, the method provides an alternative to a Maxwell construction that incorporates the impact of fluctuations on demixing. Second, and perhaps more importantly, the virtual GEMC method has pedagogical value as a tool for demonstrating the statistical nature of phase behavior and for allowing rapid exploration and analysis of fluctuations in finite systems. Our Java simulation programs can be readily adapted for use as classroom demonstrations or as numerical "experiments" in a computational laboratory. Finally, it should be straightforward to generalize the virtual GEMC method to other models and to multi-component mixtures. Future applications, for example, may explore demixing phase diagrams of ternary oil-water-surfactant mixtures.

\section{Acknowledgments}

This work was supported by the National Science Foundation under Grant No. DMR-1106331 and by the American Chemical Society Petroleum Research Fund (PRF 44365-AC7). Helpful discussions with Ben Lu are gratefully acknowledged.

[1] D. Chandler, Introduction to Modern Statistical Mechanics (Oxford, Oxford, 1987).

[2] D. Frenkel and B. Smit, Understanding Molecular Simulation, 2nd ed. (Academic, London, 2001).

[3] M. P. Allen and D. J. Tildesley, Computer Simulation of Liquids (Oxford, Oxford, 1987).

[4] D. P. Landau and R. Alben, "Monte Carlo Calculations as an Aid in Teaching Statistical Mechanics," Am. J. Phys. 41, 394-400 (1973).

[5] G. Sauer, "Teaching classical statistical mechanics: A simulation approach," Am. J. Phys. 49, 13-19 (1981).

[6] N. B. Wilding, "Computer simulation of fluid phase transitions," Am. J. Phys. 69, 1147-1155 (2001).

[7] A. Z. Panagiotopoulos, "Direct determination of phase coexistence properties of fluids by Monte Carlo simulation in a new ensemble," Mol. Phys. 61, 813-826 (1987).

[8] A. Z. Panagiotopoulos, N. Quirke, M. Stapleton, and D. J. Tildesley, "Phase equilibria by simulation in the Gibbs ensemble: alternative derivation, generalization and application to mixture and membrane equilibria," Mol. Phys. 63, 527-545 (1988).

[9] A. Z. Panagiotopoulos, "Direct Determination of Fluid Phase Equilibria by Simulation in the Gibbs Ensemble: A Review," Mol. Sim. 9, 1-23 (1992). 
[10] A. Z. Panagiotopoulos, "Gibbs Ensemble Techniques," in Observation, Prediction, and Simulation of Phase Transitions in Complex Fluids, NATO ASI Series C 460, edited by M. Baus, L. R. Rull, and J. P. Ryckaert, 463-501 (Kluwer, Dordrecht, 1995).

[11] P. G. Bolhuis, A. A. Louis, and J.-P. Hansen, "Influence of Polymer-Excluded Volume on the PhaseBehavior of Colloid-Polymer Mixtures," Phys. Rev. Lett. 89, 128302-1-4 (2002).

[12] B. Lu and A. R. Denton, "Crowding of polymer coils and demixing in nanoparticle-polymer mixtures," J. Phys.: Condens. Matter 23, 285102-1-9 (2011).

[13] B. Lu and A. R. Denton, "Phase separation of charge-stabilized colloids: A Gibbs ensemble Monte Carlo simulation study," Phys. Rev. E 75 061403-1-9 (2007).

[14] M. Mehta and D. A. Kofke, "Implementation of the Gibbs ensemble using a thermodynamic model for one of the coexisting phases," Mol. Phys. 79, 39-52 (1993).

[15] N. Metropolis, A. W. Rosenbluth, M. N. Rosenbluth, A. H. Teller, and E. Teller, "Equation of State Calculations by Fast Computing Machines," J. Chem. Phys. 21, 1087-1092 (1953).

[16] P. J. Flory, Statistical Mechanics of Chain Molecules (Wiley, New York, 1969).

[17] P. G. de Gennes, Scaling Concepts in Polymer Physics (Cornell, Ithaca, 1979).

[18] H. Gould, J. Tobochnik, and W. Christian, An Introduction to Computer Simulation Methods, 3rd ed. (Addison Wesley, 2006).

[19] Our Java simulation programs, implementing the virtual GEMC method within the Open Source Physics library, are freely available upon request.

[20] P. N. Pusey, in Liquids, Freezing and Glass Transition, session 51, ed. J.-P. Hansen, D. Levesque, and J. Zinn-Justin (North-Holland, Amsterdam, 1991).

[21] S. M. Ilett, A. Orrock, W. C. K. Poon, and P. N. Pusey, "Phase behavior of a model colloid-polymer mixture," Phys. Rev. E 51, 1344-1352 (1995).

[22] W. C. K. Poon, "The physics of a model colloid-polymer mixture," J. Phys.: Condens. Matter 14, R859-R880 (2002).

[23] S. Asakura and F. Oosawa, "Surface Tension of HighPolymer Solutions," J. Chem. Phys. 22, 1255 (1954).

[24] A. Vrij, "Polymers at interfaces and the interactions in colloidal dispersions, Pure E6 Appl. Chem. 48, 471-483 (1976).

[25] A. R. Denton and M. Schmidt, J. Phys.: Condens. Matter 14, 12051 (2002).

[26] H. N. W. Lekkerkerker, W. C. K. Poon, P. N. Pusey, A. Stroobants, and P. B. Warren, Europhys. Lett. 20, 559 (1992).

[27] S. M. Oversteegen and R. Roth, J. Chem. Phys. 122, 214502 (2005).

[28] J.-P. Hansen and I. R. McDonald, Theory of Simple Liquids, $2^{\text {nd }}$ ed. (Academic, London, 1986).

[29] M. Mehta and D. A. Kofke, "Coexistence diagrams of mixtures by molecular simulation," Chem. Eng. Sci. 49, 2633-2645 (1994). 